\title{
Development of a methodology for predicting the development of rice blast in southern Russia to improve the environmental friendliness and food security of the country
}

\author{
Dmitry Nartymov ${ }^{1, *}$, Elena Dubina ${ }^{1}$, Sergey Garkusha ${ }^{1}$, and Nikita Istomin ${ }^{1}$ \\ ${ }^{1}$ FSBSI «All-Russian Rice Research Institute», Belozerniy, 3, Krasnodar, 350921, Russia
}

\begin{abstract}
Establishing links between the conditions for the development of rice blast and the dynamics of its manifestation makes it possible to assess the behavior of the pathogen in various agroclimatic conditions. Therefore, to conduct research, we selected test sites located in one agroclimatic zone and homogeneous in variety composition. This approach allowed us to assess the degree of influence of external factors on the development of the pathogen, using different models describing this interaction. As a result of the revealed patterns, a model was determined that most accurately describes the periods of infection and the manifestation of the disease. In addition, the study set the task of the most complete coordination of the results obtained after processing the data using the model, and the data obtained as a result of observations. Thus, studies have shown that the methods used describe roughly the development processes of the Pyricularia Oryzae Cav., pathogen. However, it is necessary to rationalize the parameters of the models and consider the possibility of implementation in the territorial agro-climatic conditions of the south of Russia.
\end{abstract}

\section{Introduction}

Rice blast (causative agent - imperfect fungus Pyiricularia Oryzae Cav.) is one of the most dangerous fungal diseases in the cultivation of Oryza sativa L. around the world [4, 5]. A pathogen is a microorganism, which, by its nature, is able to adapt quickly enough to environmental conditions, both from the climate side and as a result of agrotechnological measures $[3,7]$. Therefore, in order to prevent the development of blast in a timely manner, it is very important today to monitor the disease and the development of the disease under the prevailing macroclimate, modern cultivation technologies and a large variety of rice crop, as well as to identify patterns that reflect the effect on the dynamics of the disease of cultivated plants $[8,10]$.

Establishing links between the conditions for the development of rice blast and the dynamics of its manifestation makes it possible to assess the behavior of the pathogen in

\footnotetext{
*Corresponding author: lenakrug1@rambler.ru
} 
various conditions formed by the environment and build a model for the reaction of the parameters of the disease process to environmental factors (agroclimatic conditions). Such a model can characterize the features of a pathogen population developing in a certain territory, and the model parameters and operators can be used to optimize the use of plant protection products.

To date, research work in the field of studying the dynamics of the development of rice blast is rather scattered, has a rather point-like character and it is impossible to build a noticeable trend line in solving this issue. This is largely due to the fact that the manifestation of the disease and the response of the pathogen to environmental conditions depends on the region where the population is located, the level of applied agrochemical and agrotechnological approaches, as a result, the establishment of general patterns is quite difficult. Therefore, the solution to the problem of the development of rice blast for the most part goes to the field of seed production. However, development of variety is a rather lengthy process that requires a large number of scientific studies and, as a result, takes a lot of time, while a natural change in the pathogen can minimize the resistance of the variety $[1,11]$.

Under these conditions, the dynamics of rice blast development in the period from one year to several years can become quite unpredictable. This will entail an unforeseen and irrational expenditure of resources, both material (expenses for processing plants) and intangible (loss of grain quality, decrease in the credibility of the producer). Therefore, to reduce losses, it is necessary to continuously monitor the dynamics of the conditions for rice blast development and to respond in a timely manner to indicators that contribute to the threatening development of the pathogen throughout the entire vegetation period of plants. A solution to the problem can be found in the study of the possibility of predicting the development of blast at an early stage or at the stage of spread of conidia, which can lead to infection of plants. This approach will contribute to a more rational implementation of agrochemical measures, while reducing the burden on the environment.

The environment that determines the conditions under which the pathogen develops is a physical environment, the state of which can be described using scalar mathematical parameters. The dynamics of changes in such parameters reflect the conditions that are able to describe the area of influence of external factors on the development of the pathogen. Thus, by identifying a set of conditions conducive to blast development, it is possible to construct a mathematical model of the dynamics of pathogen development, which reflects the level of reactive interaction of the microorganism and the environment. The purpose of this study is to identify the optimal development model of rice blast based on monitoring data on the dynamics of the pathogen in agroclimatic conditions that determine its behavior in the rice growing area of the Krasnodar region, the main rice producing region in the Russian Federation.

\section{Materials and methods of research}

Identification of the general patterns of interaction of the pathogen Pyricularia Oryzae Cav. with the environment is largely determined by its response to changes in agro-climatic conditions [5], herefore, within the framework of the study, test sites were selected with uniformity in varietal composition and intensity of agrotechnological measures located in one agro-climatic zone, and climate data was monitored inside this zone that affect the development of the disease. This approach makes it possible to evaluate the results obtained in terms of uniformity and repeatability.

The degree of influence of external factors on the development of microorganisms is determined by the models for predicting the dynamics of the development of the pathogen established in guidelines by V.I. Terekhov [4] and scientific works of V.G. Anikeev [1]. 
The choice of methods is due to the fact that these models make it possible to determine the conditions for the infection of plants with blast and to identify the features of the dynamics of the pathogen behavior over time. Moreover, the methods are statistical in nature and contain a mathematical implementation, which allows us to obtain comparable results. To use the techniques in research, the conventions "Method $\alpha$ " (Terekhov, 1989) and "Method $\beta$ " (Anikeev, 1990) were adopted.

To simplify the collection and processing of data, meteorological conditions were collected in a database, the structure of which allows obtaining the results of the application of the methods $\alpha$ and $\beta$ with the necessary accuracy and reliability.

\section{Results and discussion}

As a result of the studies, a number of algorithms have been developed that allow to determine the degree of pathogen reaction to changes in agroclimatic conditions in the framework of the methods $\alpha$ and $\beta$.

So, according to method $\alpha$, the algorithm may include detection of infection of plants with conidia of Pyricularia Oryzae Cav. and measuring the length of the incubation period, the end of which gives rise to the first appearance of the disease on plants. In this case, according to the method $\beta$, it is possible to determine the conditions of infection, based on weather conditions. Thus, it is possible to track the dynamics of the conditions affecting the development of the pathogen over time and obtain a diagram of the development of rice blast for test sites (Figures 1, 2).

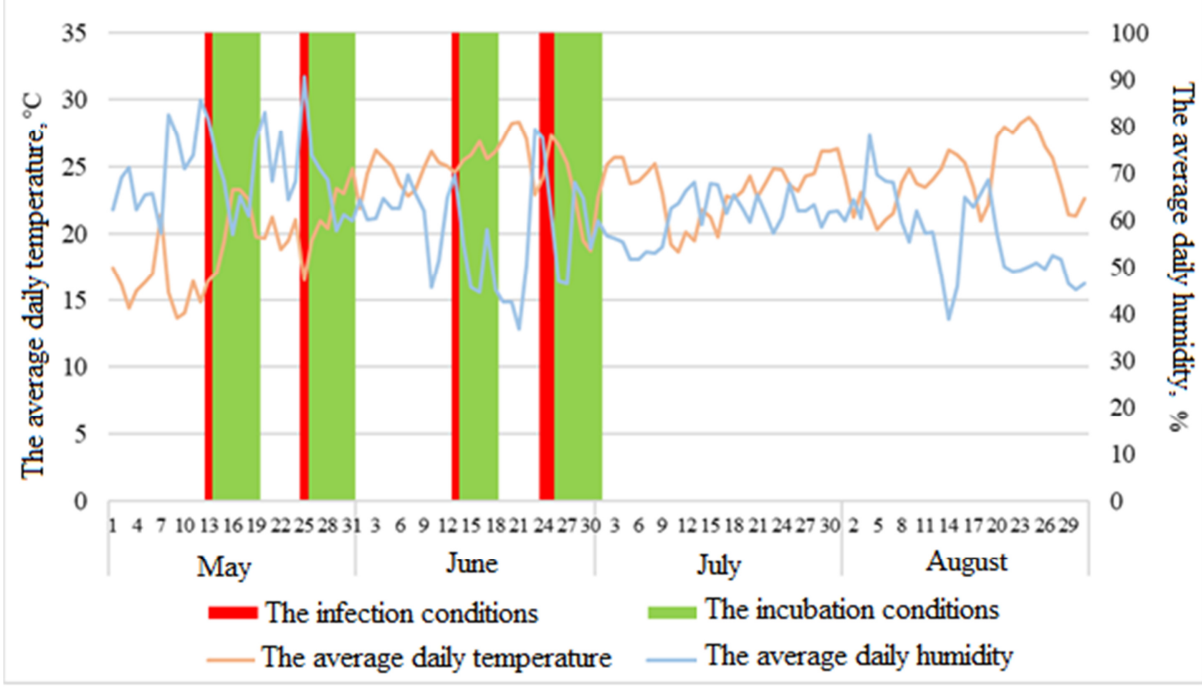

Fig. 1. Modeling the dynamics of the development of blast by the $\alpha$ method

Since, according to the chosen approaches, the test sites are located under homogeneous conditions (meteorological conditions, agricultural technology, variety resistance), the time for the manifestation of traits of plant diseases did not vary much and did not exceed 24 48 hours. This made it possible to compare the results of the algorithms and the traits of the manifestation of plant disease. So, the first manifestations of blast on the leaf plate of plants on test sites were noticed on June 27, and on July 5 there was a need to carry out the first treatment of plants. From the diagram (Figure 1) constructed by the $\alpha$ method, it is seen that conditions favorable for the development of plant disease arose between June 24 and 
30, however, such periods were also observed earlier, but no emergence of the disease was observed. This is due to the fact that in the early periods local infections occur that increase the spore infestation of plants slightly and are not able to cause epiphytotics. In addition, the average daily temperature greatly inhibits the development of the pathogen. However, contamination with spores increases (...). As a result of this, under favorable conditions, the probability of infection of a large number of plants increases.

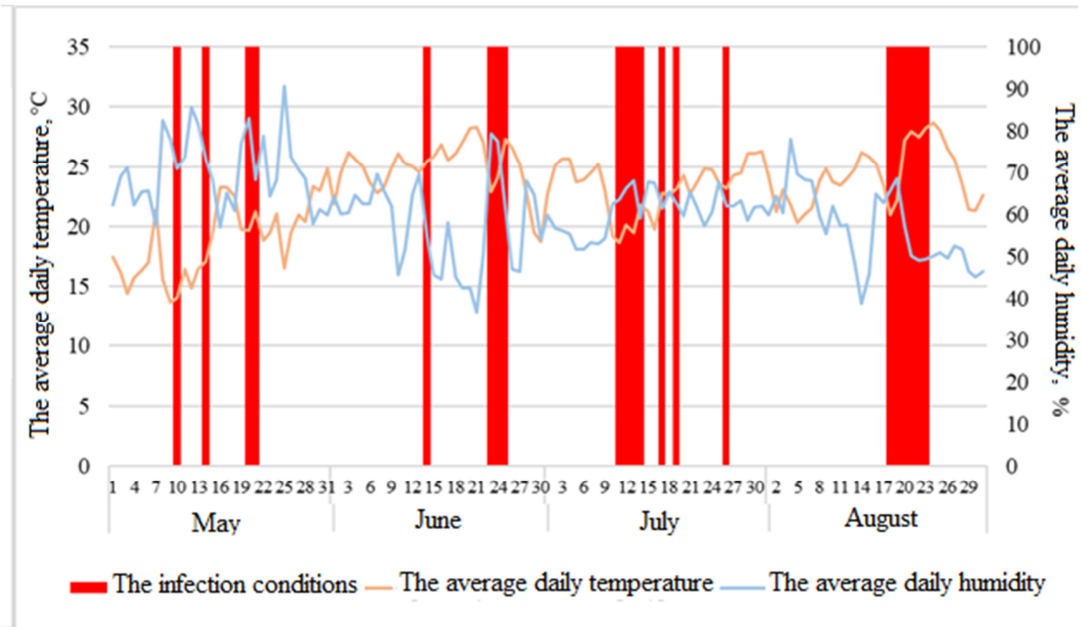

Fig. 2. Modeling the dynamics of the development of blast by the $\beta$ method

In contrast to the method $\alpha$, the dynamics of the main indicators characterizing the degree of influence of meteorological conditions on infection with blast, constructed according to the method $\beta$, is practically inconsistent with the appearance of the disease on June 27, July 5 and August 3, however, the detection of infection periods is quite close to the results of the method $\alpha$. Perhaps the application of this method requires the adjustment of parameters and further study of methods for its implementation.

Simulation of the dynamics of the development of Pirycularia Oryzae Cav. pathogen, based on an analysis of meteorological data for 2018, is shown in Figure 3. It can be seen from the figure that the detection of infection conditions determined by the two models $\alpha$ and $\beta$ is correlated, and the detection difference is no more than two days. In addition, the definition of probability in a given period is at the same level. The obtained data vividly illustrate the convergence of the simulation results by different methods, and the detection difference can be eliminated by adjusting the parameters of the models, which requires a deeper analysis and study of the models for their application in the south of Russia.

It is important to note that the meteorological data that were used in the studies are determined by state monitoring at meteorological stations located at control points. These stations are located at some distance from the test sites selected for the study, however, such an arrangement, on the one hand, introduces a fraction of the error due to the heterogeneity of the atmosphere, and on the other hand makes it possible to relate the data of continuous monitoring of meteorological data and the dynamics of the development of the pathogen. With proper modeling, this approach will allow monitoring the dynamics of blast development, as well as make both medium and long-term prognosis of pathogen development based on prediction of meteorological conditions.

In addition, the use of state monitoring data ensures the reliability of indicators and the stability of observations. The development of a forecasting model using these data is most 
rational, since the parameters of the model are confirmed by a reliable source, and the model can be implemented with similar data in order to implement an integrated system for monitoring and predicting rice blast in the south of Russia.

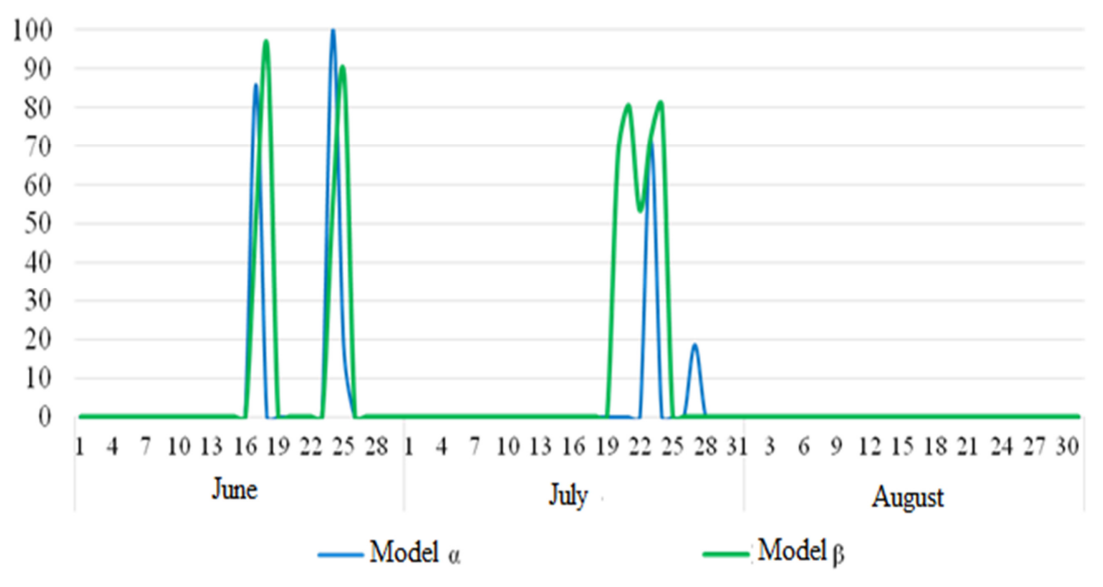

Fig. 3. Modeling the dynamics of blast development in 2018

In addition, when monitoring meteorological data, the development of specialized devices that allow for monitoring the state of the environment is of great importance. The installation of such devices in the agroclimatic zone will make it possible to control the dynamics of changes in the scalar parameters of the environment in which the pathogen develops, and to determine the degree of its reaction to changes in conditions more accurately, and to make the forecast level higher.

\section{Conclusions}

Studies have shown that the most acceptable results describing the dynamics of the development of rice blast are given by the application of the method $\alpha$, developed and proposed by V.I. Terekhov. The method involves the detection of conditions of infection of plant organs with conidia of the microorganism Pyricularia Oryzae Cav. and calculating the incubation period of the pathogen, the end of which indicates the first manifestations of damage. This method will allow you to most rationally organize the periods of application of plant protection products in agricultural production.

The parameters of the method require rational implementation in the conditions of the cultivation territory and, as a result, further study. Optimization of these algorithms will make it possible to monitor the epiphytotic state of rice-growing areas and use them in rationalizing the rice production process in the South of Russia, since the timely use of protective equipment significantly reduces the chemical load on the production result and the environment.

\section{References}

1. V.G. Anikeev, Abstract of Ph.D., 28 (1990)

2. I.A. Kostenko, Abstract of Ph.D., 24 (2006)

3. Yu.M. Morozov, Abstract of Ph.D. 26 (1991)

4. E.V. Dubina, P.I. Kostylev, M.G. Ruban, Yu.V. Aniskina, I.A. Shilov, N.S. Velishaeva, L.V. Esaulova, 6 (54), 29-35 (2017) 
5. V.I. Terekhov, Agropromizdat, 24 (1989)

6. V.A. Todirash, T.F. Tretyakova, V.I. Foksha, I.D. Mangul, V.I. Stratu, Bulletin of plants protection, 1, 50-53 (2008)

7. T.V. Dudchenko, Pl. prot. and quar., 8, 29-32 ( 2017)

8. N.A. Evsyukov, Yu.G. Sokolov, Pl. prot. and quar., 10, 40-41 (2010)

9. R.Yu. Danilov, V.Ya. Ismailov, V.A. Tretyakov, O.Yu. Kremneva, Yu.V. Shumilov, A.A. Rizvanov, V.V. Krivoshein, I.A. Kostenko, Mat. of the II All-Russian entific conference with international participation "The use of remote sensing of land in agriculture", 367-374 (2018)

10. E.V. Dubina, M.G. Ruban, Yu.V. Aniskina, I.A. Shilov, N.S. Velishaeva, P.I. Kostylev, Yu.A. Makukha, D.A. Pischenko, Ach. of Sc. and Tech. of AIC, 32/10, 19$23(2018)$

11. E.V. Dubina, Z.M. Mukhina, E.M. Kharitonov, V.N. Shilovskiy, E.S. Kharchenko, L.V. Esaulova, N.N. Korkina, E.P. Maximenko, I.B. Nikitina, Rus. J. of Gen., 51/8, 752-756 (2015)

Criteria of authorship. The authors of the article confirm that D.V. Nartymov has $85 \%$ of authorship rights, EV Dubina - 5\%, Garkusha S.V. - 5\%, Istomin N.K. - 5\% and are equally responsible for plagiarism.

Conflict of interest. The authors declare no conflict of interest.

The studies were carried out as part of the research of State task No. 0494-2019-0001 "Information forecasting of the development of rice and vegetable diseases and development of new genetic resources using modern biotechnological approaches" with financial support from the Russian Ministry of Education and Science. 